\title{
Impacto de la globalización en las estrategias de negocios en las empresas ecuatorianas.
} Impact of globalization on business strategies in Ecuadorian
companies.

\author{
Tec. María Gabriela Flores ${ }^{1}{ }^{*}$, Lic. Jackeline Dayana Flores ${ }^{2}$ \\ 1.* Tecnólogo en Administración Mención Mercadotecnia, Universidad Centroccidental Lisandro Alvarado, Barquisimeto, \\ Venezuela. Email: maria.flores@ucla.edu.ve ORCID: https://orcid.org/0000-0001-8985-013X
}

2. Licenciada en Educación Mención Arte, U.E. Adventista Profesor “Clímaco Girón”, Barquisimeto, Venezuela. Email: jackelinef07@gmail.com ORCID: https://orcid.org/0000-0002-9045-9370

Destinatario: maria.flores@ucla.edu.ve

Recibido: 30/ febrero/2021 Aceptado: 30/marzo/2021 Publicado: 30/abril/2021

Como citar: Flores, M. G., \& Flores , J. D. (2021). Impacto de la globalización en las estrategias de negocios en las empresas ecuatorianas. Revista E-IDEA Journal of Business Sciences, 3(11), 1-11. https://doi.org/10.53734/eidea.vol3.id88

\begin{abstract}
Resumen: Las organizaciones hoy en día deben enfrentar numerosos cambios producto de la globalización y la dinámica existente en los procesos tanto internos como externos de las empresas. Deben procesar grandes cantidades de información para poder lograr cumplir sus objetivos y metas, esto se logra a través de aspectos como la innovación, creatividad, crecimiento, competitividad y la toma de decisiones El objetivo general de la investigación se basa en analizar el impacto de la globalización en las estrategias de negocio de las empresas ecuatorianas. La metodología utilizada se basa en un diseño bibliográfico de tipo documental. Como conclusion se puede mencionar que La globalización es pieza fundamental en el desarrollo de los países, sin embargo muchos poseen limitantes que no permiten el desarrollo a cabalidad de nuevas estrategias para lograr llegar al nivel máximo de sus capacidades, tal es el caso de Ecuador que a pesar de los grandes pasos logrados aun está en proceso de desarrollo para implementar la tecnología necesaria y lograr mayores beneficios económicos así como estabilidad económica, política y social Son variados los cambios que surgen producto de la globalización y a su vez permiten que las empresas logren adaptar tecnologías en sus procesos productivos, esto es señal de que su personal debe estar preparado intelectualmente para el manejo de las nuevas tecnologías y poder aprovechar al máximos estas innovaciones que darán fruto trayendo consigo beneficios económicos, rentabilidad, eficiencia y aprovechamiento de los recursos existentes.
\end{abstract}

Palabras Clave: innovación, globalización, estrategias, negocios, empresas, rentabilidad.

Abstract: Organizations today must face numerous changes as a result of globalization and the existing dynamics in both internal and external processes of companies. They must process large amounts of information to be able to achieve their objectives and goals, this is achieved through aspects such as innovation, creativity, growth, competitiveness and decision-making. The general objective of the research is based on analyzing the impact of the globalization in the business strategies of Ecuadorian companies. The methodology used is based on a bibliographic design of a documentary type. As a conclusion, it can be mentioned that globalization is a fundamental piece in the development of countries, however many have limitations that do not allow the full development of new strategies to achieve the maximum level of their capacities, such is the case of Ecuador that Despite the great steps achieved, it is still in the process of development to implement the necessary technology and achieve greater economic benefits as well as economic, political and social stability. The changes that arise as a result of globalization are varied and in turn allow companies to adapt technologies in their production processes, this is a sign that your staff must be intellectually prepared to handle new technologies and be able to make the most of these innovations that will bear fruit, bringing with them economic benefits, profitability, efficiency and use of existing resources.

Keywords: innovation, globalization, strategies, business, companies, profitability. 


\section{INTRODUCCIÓN}

$\mathrm{L}$

as organizaciones hoy en día deben enfrentar numerosos cambios producto de la globalización y la dinámica existente en los procesos tanto internos como externos de las empresas. Deben procesar grandes cantidades de información para poder lograr cumplir sus objetivo y metas, esto se logra a través de aspectos como la innovación, creatividad, crecimiento, competitividad y las tomas de decisiones (Cordero-Guzmán y Rodriguez- López, 2017). Cada uno de estos aspectos son fundamentales para lograr mejoras en la calidad de los productos o servicios y se logren buenos márgenes de ganancias.

Asimismo, la información que se maneje debe ser bien estructurada, de manera que pueda llegar a cada uno de los departamentos necesarios para ejecutar las actividades correspondientes de forma eficiente, a través del uso de herramientas necesarias que logren establecer diferencias competitivas con el resto de las empresas.

Estas diferencias frente a la competencia se logran a través del uso de estrategias de negocios que permitan un desarrollo integral de las actividades, usando ciencia, tecnología e innovación que permitirá mantenerse en un dinamismo constante logrando un crecimiento tanto económico como organizacional y sea capaz de enfrentar los efectos causados por la globalización existente en el mundo (Jarrín, Villalba, y Paredes, 2019). Cabe destacar que estos procesos de innovación no siempre son ejecutados por las empresas por diversas razones, lo que provoca limitaciones en muchas ocasiones dentro de las organizaciones, sin embargo, pese a las limitantes los efectos de la globalización empuja a que las empresas tomen conciencia y noten la necesidad existente en un cambio de pensamiento, métodos y procesos de actualización permitiendo generar ideas que logren descubrir nuevas estrategias y lograr estar a la par con muchas empresas de talla internacional. (Naranjo y Pastor, 2009).

Parte de las nuevas estrategias se centran en el uso de nuevas tecnologías denominadas, TIC`s que son parte fundamental para la comunicación entre el comercio y la tecnología en general, logrando un mercado más digitalizado adaptándose a este mundo tan cambiante. De la misma manera, es necesario que las empresas puedan comunicarse a través de la tecnología y por medio de ella ofrezcan sus productos y/o servicios y también sirva como método para obtener los beneficios económicos el cual es el principal fin de cada una de las empresas (Pesántes-Calva, Romero-Correa, y González-Illescas, 2020).

Estas tecnologías de la Información y Comunicación (TIC) son desarrolladas luego de muchos estudios científicos que dio paso en la informática a avances significativos que permiten la fluidez de la comunicación desde cualquier parte del mundo, en este sentido Cabero 1998 citado por Belloch (2012) menciona que las TIC es definida como:

En líneas generales podríamos decir que las nuevas tecnologías de la información y comunicación son las que giran en torno a tres medios básicos: la informática, la microelectrónica y las telecomunicaciones; pero giran, no sólo de 
forma aislada, sino lo que es más significativo de manera interactiva e interconexionadas, lo que permite conseguir nuevas realidades comunicativas (Cabero 1998, p. 198 Citado por Belloch 2012).

Son diversos los dispositivos electrónicos que permiten el uso de las TICS como la televisión, el teléfono, el ordenador entre otros, pero los que más representan a la sociedad son los equipos de computación donde pueden utilizar diversas aplicaciones desde sus hogares u oficinas y por medio de ellas ejecutar operaciones electrónicas.

A su vez Belloch (2012) menciona algunas características de las TIC y son las mostradas en la Tabla 1.

\section{Tabla 1}

Características de las TIC:

\begin{tabular}{|c|c|}
\hline CARACTERISTICAS & CONCEPTOS \\
\hline Información multimedia & $\begin{array}{l}\text { El proceso y transmisión de la información abarca todo tipo } \\
\text { de información: textual, imagen y sonido, por lo que los } \\
\text { avances han ido encaminados a conseguir transmisiones } \\
\text { multimedia de gran calidad. }\end{array}$ \\
\hline Interactividad & $\begin{array}{l}\text { La interactividad es posiblemente la característica más } \\
\text { importante de las TIC para su aplicación en el campo } \\
\text { educativo. Mediante las TIC se consigue un intercambio de } \\
\text { información entre el usuario y el ordenador. Esta } \\
\text { característica permite adaptar los recursos utilizados a las } \\
\text { necesidades y características de los sujetos, en función de la } \\
\text { interacción concreta del sujeto con el ordenador }\end{array}$ \\
\hline Interconexión & $\begin{array}{l}\text { La interconexión hace referencia a la creación de nuevas } \\
\text { posibilidades tecnológicas a partir de la conexión entre dos } \\
\text { tecnologías. Por ejemplo, la telemática es la interconexión } \\
\text { entre la informática y las tecnologías de comunicación, } \\
\text { propiciando con ello, nuevos recursos como el correo } \\
\text { electrónico, los IRC, etc. }\end{array}$ \\
\hline Inmaterialidad & $\begin{array}{l}\text { En líneas generales podemos decir que las TIC realizan la } \\
\text { creación (aunque en algunos casos sin referentes reales, } \\
\text { como pueden ser las simulaciones), el proceso y la } \\
\text { comunicación de la información. Esta información es } \\
\text { básicamente inmaterial y puede ser llevada de forma } \\
\text { transparente e instantánea a lugares lejanos. }\end{array}$ \\
\hline $\begin{array}{c}\text { Mayor Influencia sobre los procesos } \\
\text { que sobre los productos }\end{array}$ & $\begin{array}{l}\text { Es posible que el uso de diferentes aplicaciones de la TIC } \\
\text { presente una influencia sobre los procesos mentales que } \\
\text { realizan los usuarios para la adquisición de conocimientos, } \\
\text { más que sobre los propios conocimientos adquiridos. }\end{array}$ \\
\hline
\end{tabular}

Fuente: (Belloch, 2012)

Estas nuevas estrategias tecnológicas permiten a las empresas ecuatorianas estar al nivel de empresas de otros países y sean capaces de lograr mantenerse en el tiempo, por lo que fortalecer las TIC's les permite lograr estar a la par de los avances acelerados de este 


\section{E-IDEA}

J OURNAL OF B USINESS SCIENCES

mundo digitalizado. De esta manera, Zúñiga, Marchán y López (2020) indica que las TIC's han creado un "cambio en la manera de vivir de la sociedad", y ha provocado una nueva manera de comunicarse, es así como el comercio electrónico se convirtió en una estrategia moderna e innovadora permitiendo a los usuarios que la utilizan adquirir productos de acuerdo a sus necesidades agilizando los procesos de entrega y pago.

Es así que el comercio electrónico según Gascón 2018 citado por Zúñiga et al. (2020) es definido como:

Aquellos procesos de negocios que se realizan por medio de una nube informática, en la que se efectúan compras a través de dispositivos electrónicos con acceso a internet, es decir, se trata de un comercio en donde se gestiona cobros y pagos mediante medios electrónicos (pág. 3)

Surgieron intentos de este tipo de tecnologías a finales del siglo XIX en Estados Unidos, posteriormente en los años 70 surge la revolución del internet y nace una interacción entre las personas por todo el mundo, aparece en los 90's la World Wide Web permitiendo que el comercio se maneje por medio del internet donde se da inicio a la interacción entre diversas redes informáticas que permiten divulgar, informar y recibir información que permite transacciones comerciales. Posteriormente las empresas comenzaron a utilizar esta tecnología que le permitió lograr la venta y compra de productos y/o servicios (Zúñiga et al. 2020).

En este sentido Pesántes-Calva et al. (2020) menciona que una de las innovaciones del comercio electrónico es el B2B y es definido como

Transacción económica efectuada entre empresas que utilizan la internet y diversas tecnologías con la finalidad de conseguir múltiples beneficios, entre los cuales podemos mencionar los siguientes: mayor eficiencia, aumento de ventas, mejoras en las relaciones con los clientes y mayor penetración en el mercado (pág. 5)

Este tipo de comercio electrónico es poco usado ya que predomina el uso del C2C (operación comercial entre consumidores) o B2C (venta de empresa a consumidor final), sin embargo es importante que este tipo de comercio electrónico sea implementado por las empresas en alianza con el gobierno y otras instituciones pues es una estrategia que permitirá a las organizaciones del país lograr ser competente frente a este mundo cambiante y acelerada (Pesántes-Calva, Romero-Correa y González-Illescas, 2020).

Los negocios emprendedores de Ecuador han tomado la iniciativa de apostar a la tecnología pues es un puente que permite la internacionalización, llegando a lugares y creando nuevas alianzas comerciales con otras empresas que permiten lograr mayores beneficios económicos, en este sentido, Jurado (2019), indica que en el año 2018 se reunieron en Quito más de mil expertos en negocios por internet y celebraron la $7 \mathrm{ma}$ versión del eCommerce Day Ecuador, en este evento muchos emprendedores y personal 
ejecutivo de diversas empresas privadas participaron en talleres acerca del manejo de los negocios a través de la internet tanto a nivel regional como nacional, capacitándose para hacer frente a los constantes cambios y desafíos presentes dentro de la economía producto de la globalización, que permita a las empresas crecer y lograr rentabilidad.

En este sentido el objetivo general de esta investigación es analizar el impacto de la globalización en las estrategias de negocio de las empresas ecuatorianas. La metodología utilizada se basa en un diseño bibliográfico de tipo documental.

\section{METODOS}

La metodología empleada en esta investigación se basa en un diseño bibliográfico de tipo documental a través de una revisión sistemática de artículos científicos indexados en bases de datos científicas con verificable rigor académico basados en temas de Globalización, estrategias de negocios, impacto de la globalización en Ecuador, se concreta exclusivamente en la recopilación de información de diversas fuentes, con el objeto de organizarla describirla e interpretarla de acuerdo con ciertos procedimientos que garanticen confiabilidad y objetividad en la presentación de los resultados (Palella Stracuzzi y Martins Pestana, 2010).

\section{RESULTADOS Y DISCUSIÓN}

\section{La globalización. Aspectos relevantes}

Los impactos que genera la globalización en los países son de gran envergadura, tanto a nivel político, social, económico, cultural y tecnológico, un ejemplo de esto es la influencia cultural que genera este proceso causando en ocasiones niveles de transculturización inclusive preocupantes pues la sociedad olvida lo propio para adoptar creencias y culturas de otros lugares lejanos. En este sentido Burgos (2017), menciona que estas mezclas de naciones han cambiado hasta la forma de vivir, los niveles de consumo, de manera que los países cada día se encuentran más interconectados.

La globalización es proveedora de aspectos positivos y negativos dentro de cada uno de los países, según Vallejo (2011), esta incrementa los beneficios económicos, la productividad y la rentabilidad de las empresas, sin embargo no siempre ocurre esto en los países en desarrollo o aquellos países mas pobres, y a pesar de estas limitantes, no puede dejarse a un lado pues y deber de estos países afrontar sus falencias y crear una nueva cultura dentro de su sociedad que comprenda que la globalización es parte del mundo.

Muchas fallas presentes en los países desarrollados es producto del mal manejo de estrategias y métodos para adaptarse a los efectos de la globalización, que impide el fortalecimiento económico y debilitamiento de los procesos productivos. 


\section{E-IDEA}

J OURNAL OF B USINESS SCIENCES

Uno de los aspectos positivos que posee la globalización es la inversión extranjera, donde empresas de otros países apuestan por invertir en el país, pues con ella, según lo menciona Burgos (2017, permite crear nuevas fuentes de trabajo, que se traduce en bienestar social. Visto de este punto de vista un trabajo mancomunado, permite eficiencia que puede llegar a resultar en beneficios mutuos y logro de objetivos que permiten el fortalecimiento de las organizaciones, en este orden de ideas Montilla, Rodríguez, Mora, y Mera (2018), indica que los cambios generados por la globalización son significativos, pero cada empresa son las encargadas de aprovechar sus capacidades para lograr los mejores beneficios para todo el grupo de trabajo.

\section{Características y elementos que conforman la globalización}

En un mundo tan cambiante y con un mercado tan variado, la economía es fundamental para conocer cada aspecto importante en cada una de las actividades comerciales de las organizaciones que prestan algún tipo de servicio o producto, para lograr un equilibrio entre los agentes económicos y los compradores de manera que el sistema económico sea positivo y beneficioso para todos los que intervienen en cada proceso (Moreno, 2018).

La globalización surge por la necesidad imperante de la ampliación de las exigencias de la sociedad en el mundo, y la gran variedad de transacciones económicas existentes por lo que esta trascendencia es más evidente cuando surge la necesidad de que las instituciones mejoren los procesos de mercantilización y se encarguen de cubrir los gastos que implican la innovación tecnológica que permita realizar las gestiones correspondientes para la venta/adquisición de bienes prestación de servicios. La globalización ha permitido que hasta los entes gubernamentales en el caso de Ecuador y algunos países de Latinoamérica logren adaptarse a este tipo de e-Gobernanza para agilizar los procesos y mejorar la experiencia de sus ciudadanos al momento de realizar gestiones en los entes públicos, minimizando la burocracia y muchos aspectos que traen consigo las malas gestiones (Moreno, 2018).

Es fundamental que la innovación y las estrategias de negocios estén siempre presentes en las organizaciones y en base a esto Wang y Ahmed 2004 citado por Acosta et al. (2018) muestra en la Tabla 2 algunos conceptos de Innovción desde un enfoque contemporaneo descrito por algunos importantes autores y se muestra a continuación:

\section{Tabla 2}

La Innovación desde un enfoque contemporáneo:

\begin{tabular}{|c|l|}
\hline AUTORES & \multicolumn{1}{|c|}{ PROPUESTA } \\
\hline Schumpeter (1934) & $\begin{array}{l}\text { Lo define como una nueva forma de producción, abarca la } \\
\text { creación de un nuevo bien o proceso, la gestión de la } \\
\text { organización, su estructura, apertura de nuevos mercados, entre } \\
\text { otros. }\end{array}$ \\
\hline Schmookle (1966) & $\begin{array}{l}\text { Surge tras la necesidad de solucionar un problema, las ideas } \\
\text { creativas e innovadoras responden a una demanda originada en } \\
\text { el entorno. }\end{array}$ \\
\hline
\end{tabular}

Flores, M. G., \& Flores, J. D. (2021). Impacto de la globalización en las estrategias de negocios en las empresas ecuatorianas. E-IDEA Journal of Business Sciences, 3(11), 1-11. https://doi.org/10.53734/eidea.vol3.id88. 
R E V I S T A

\begin{tabular}{|c|c|}
\hline Sherman (1981) & $\begin{array}{l}\text { Ideas originadas tras identificar una necesidad, dando como } \\
\text { resultado la invención de nuevos productos, procesos o técnicas } \\
\text { para lograr éxito en el mercado. }\end{array}$ \\
\hline Nelson y Winter (1982) & $\begin{array}{l}\text { Consiste en implementar nuevos diseños a los productos, } \\
\text { procesos o procedimientos ya establecidos en las organizaciones } \\
\text { con fines de crear mejoras enfatizando en la incertidumbre como } \\
\text { un riesgo por el cambio. }\end{array}$ \\
\hline Kanter (1984) & $\begin{array}{l}\text { La innovación contempla el cambio organizacional y los procesos } \\
\text { de aprendizaje para impulsar los cambios y actualizaciones. }\end{array}$ \\
\hline $\begin{array}{l}\text { Cardinal, Alessandri y Turner } \\
\text { (2001) }\end{array}$ & $\begin{array}{l}\text { Visualiza la innovación como un proceso que involucra aspectos } \\
\text { técnicos, físicos y actividades centrales basadas en el } \\
\text { conocimiento, los mismos que permiten crear rutinas } \\
\text { organizacionales para el desarrollo de productos. }\end{array}$ \\
\hline Herkema (2003) & $\begin{array}{l}\text { La innovación es esencialmente un proceso de conocimiento } \\
\text { orientado a crear nuevas ideas para el desarrollo de soluciones } \\
\text { comerciales. }\end{array}$ \\
\hline Arraut (2008) & $\begin{array}{l}\text { Transformación de la estructura interna para la mejora del } \\
\text { control y gestión organizacional, implementación de } \\
\text { herramientas tecnológicas para la optimización de procesos y } \\
\text { reducción de costos. }\end{array}$ \\
\hline Cano y Sánchez (2014) & $\begin{array}{l}\text { Uso de las capacidades y habilidades del recurso humano, y } \\
\text { tecnología en la cadena de valor con el fin de mejorar el proceso } \\
\text { de producción. }\end{array}$ \\
\hline OECD y Eurostat $(2006,2018)$ & $\begin{array}{l}\text { Es la creación o mejoramiento de un producto, proceso o a su vez } \\
\text { la combinación de ambos. Así como también el uso de modernas } \\
\text { técnicas para la gestión y la comercialización. }\end{array}$ \\
\hline
\end{tabular}

Fuente: Wang y Ahmed 2004 citado por Acosta et al. (2018)

Otra característica importante de la globalización según lo indica Acosta, Yagual, y Coronel (2018), es que ha permitido que la información y comunicación fluyan en forma vertiginosa a través de los diversos medios digitales. Las empresas privadas y el sector público se han dado a la tarea de implementar avances tecnológicos que permite generar negocios utilizando modelos y estrategias de negocios diferentes, combinando ciencia, conocimiento y tecnología para lograr el cumplimiento de sus metas.

Estos cambios digitales permiten a las empresas llegar a nuevos mercados, generar ingresos a través de nuevas fuentes de innovación, logrando mayor competitividad apoyados con el mundo digital. Estos nuevos desafíos tecnológicos deben ser implementados en toda Latinoamérica de manera que puedan lograr avanzar económicamente, y dejar de depender solo de ciertos rubros.

De manera más reciente han surgido otras innovaciones como el blockchain, que según Hernández (2017) citado por Acosta et al. (2018), es:

Un libro contable de transacciones controlado y monitoreado por varias fuentes de forma simultánea siendo sus características ser distribuido, público, transparente, encriptado e inmutable. Esta disrupción tiene un gran 


\section{E-IDEA}

J OURNAL OF BUSINESS SCIENCES

potencial incluso de reemplazar a instituciones financieras. Entre sus aplicaciones potenciales están las microfinanzas, las remesas y pagos internacionales, registros digitales, seguimientos, contratos y las donaciones entre pares (pág. 36)

Asimismo, surgen nuevas tendencias y retos, y GREYGROUP (2016) citado por Acosta et al. (2018) a través de la Figura 1 las muestra:

\section{Figura 1}

Nuevas Tendencias Digitales:

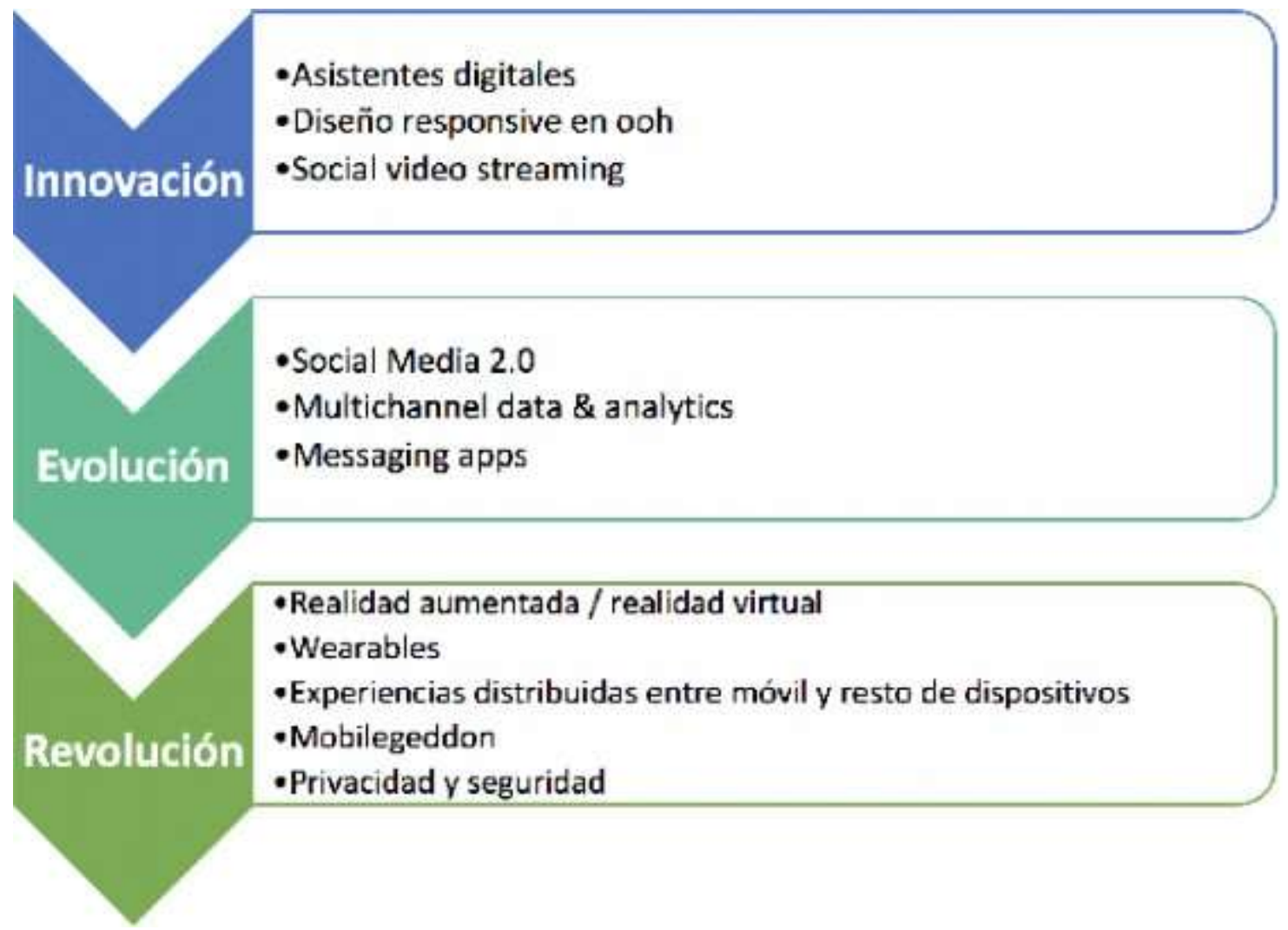

Fuente: GREYGROUP (2016) citado por Acosta et al. (2018)

En este sentido muchas empresas de gran envergadura y otras empresas ecuatorianas se han dado a la tarea de implementar sistemas integrados en primer lugar los ERP (Entreprise Resource Planning), al igual que las entidades bancarias que cuentan con bases de datos y estructuras informáticas que les permiten realizar transacciones con mayor privacidad y con menores fallas.

Todos estos avances han permitido que surja la industria 4.0 o llamada la cuarta revolución industrial implementada en Ecuador permitiendo que sea más fuerte la digitalización mejorando en gran medida los aspectos sociales, comerciales y económicos, en todos los niveles, un ejemplo de esto es el indicado por Bravo y Martillo (2019), donde 
mencionan que el Ministerio de Industria y Productividad (MIPRO) ha creado una plataforma digital que permite realizar procedimientos para detectar faltas en los servicios $\mathrm{y}$ tomar decisiones que ayuden a los sectores productivos, y esto es fundamental para el desarrollo del país. Es importante mencionar que si bien ya Ecuador está implementando estas nuevas tecnologías aún debe afrontar retos y limitaciones existentes.

\section{CONCLUSIONES}

La globalización es pieza fundamental en el desarrollo de los países, sin embargo, muchos poseen limitantes que no permiten el desarrollo a cabalidad de nuevas estrategias para lograr llegar al nivel máximo de sus capacidades, tal es el caso de Ecuador que a pesar de los grandes pasos logrados aún está en proceso de desarrollo para implementar la tecnología necesaria y lograr mayores beneficios económicos, así como estabilidad económica, política y social.

Son variados los cambios que surgen producto de la globalización y a su vez permiten que las empresas logren adaptar tecnologías en sus procesos productivos, esto es señal de que su personal debe estar preparado intelectualmente para el manejo de las nuevas tecnologías y poder aprovechar a los máximos estas innovaciones que darán fruto trayendo consigo beneficios económicos, rentabilidad, eficiencia y aprovechamiento de los recursos existentes. 


\section{E-IDEA}

J OURNAL OF BUSINESS SCIENCES

\section{REFERENCIAS BIBLIOGRÁFICAS}

Acosta, M., Yagual, A., \& Coronel, V. (2018). Perspectivas de la economía digiital en Latiinoamérica: Caso Ecuador. 3C Empresa: Investigación y Pensamiento Crítico 7(3) , 28-43.

Belloch, C. (2012). Las Tecnologías de la Información y Comunicación en el aprendizaje. Departamento de Métodos de Investigación y Diagnóstico en Educación. Universidad de Valencia.

Bravo, K., \& Martillo, K. (2019). Economia Circular y la uindustria 4.0 como estrategia del comercio internacional en el Ecuador. Guayaquil, Ecuador: Trabajo especial de grado de la Universidad de Guayaquil para optar al título de Ingeniera en Comercio Exterior.

Burgos, A. (2017). ¿Qué ventajas y desventajas tiene la globalización en el desarrollo de un pais? Expresiones, Revista Estudiantil de Investigación 4(8) , 67-69.

Cordero-Guzmán, D., \& Rodríguez-López, G. (2017). La inteligencia de negocios: una estrategias de gestion de las empresas productivas. Revista Ciencia UNEMI 10(23) , 40-48.

Jarrín, J., Villalba, G., \& Paredes, M. (2019). ¿Por qué las empresas ecuatorianas innovan? Revista Compendium: Cuadernos de Economía y Administración 6(1) , 15-27.

Jurado, P. (2019). Comercio Electrónico en Ecuador. Revista Observatorio de la Economía Latinoamericana , 1-19.

Montilla, A., Rodríguez, H., Mora, L., \& Mera, B. (2018). Retos de la gerencia en el marco de la globalizaciión y el desarrollo sustentable. Revista Pertinencia Académica , 77- 88. 
Moreno, A. (2018). El BINOMIO ECONOMIA-TECNOLOGIA Y SU RELACION CON LA GLOBALIZACION ECONOMICA. UNA ESPECIAL REFERENCIAL AL GOBIERNO ELECTRONICO. TABLA DE CONTENIDOS. Revista Jurídica de la

Universidad Católica de Santiago de Guayaquil , 110-118.

Naranjo, F., \& Pastor, B. (2009). La globalización en el ecuador y su influencia en la búsqueda de nuevas estrategias de mercado en la industria de las bebidas gaseosas. Informe.

Palella Stracuzzi, S., \& Martins Pestana, F. (2010). Metodologia de la Investigación Cuantitativa. Carcas, Venezuela: FEDUPEL, Fondo Editorial de la Universidad Pedagógica Experimental Libertador.

Pesántes-Calva, A., Romero-Correa, J., \& González-Illescas, M. (2020). Comercio electrónico B2B como estrategia competitiva en el comercio internacional: Desafios para Ecuador. INNOVA Research Journal 5(1) , 72-93.

Vallejo, M. (2011). La importancia de las relaciones internacionales para la integración económica y globalización del Ecuador. Guayaquil, Ecuador: Trabajo especial de La Universidad de Guayaquil, para optar al título de Magister en Ciencias Internacionales.

Zúñiga, E., Marchán, M., \& López, J. (2020). Modelo de Negocio Ecommerce en Ecuador. Investigación académica 1(2) , 38-52. 